\title{
Optimisation de l'élevage des pieds-mères de Douglas (Pseudotsuga menziesii (Mirb.)Franco) pour la multiplication végétative en vrac
}

\author{
Michel Verger*a, Céline Le Pichon ${ }^{\mathrm{b}}$, Michel Cazet ${ }^{\mathrm{b}}$ \\ a Inra, station d'amélioration des arbres forestiers, 45160 Ardon, France; \\ ${ }^{b}$ Cemagref, division ressources génétiques et plants forestiers, domaine des Barres, \\ 45290 Nogent/Vernisson, France
}

(Reçu le 12 mai 1997 ; accepté le 1er avril 1998)

\begin{abstract}
Study of the optimal stock-plant management of Douglas-fir (Pseudotsuga menziesii (Mirb.) Franco) for bulk vegetative propagation. The French breeding program of Douglas-fir produces seeds of very high genetic value (half or full-sib families) but in insufficient quantity to provide an important source of reforestation planting stock. Bulk vegetative propagation offers the possibility to amplify the reforcstation potential of these seed lots. Two strategies are currently being explored: a) selection of families for their ability for bulk vegetative propagation [31]; and b) improvement of the production system to enable a cheap stock-plant management. Three experiments were conducted between 1992 and 1997 at Orleans (Inra) and Nogent/Vernisson (Cemagref) to investigate different methods of managing stock-plants: the effects of different growing environments, of artificial and natural lighting and of pruning and the behaviour of top and intermediate-cuttings were compared. The best management consists in a first crop of cuttings on 2-year-old stock-plants grown on nursery soil with a plastic mulch and under a $50 \%$ white shaded tunnel. In this case, the multiplication rate (number of rooted cuttings per stock-plant) reaches 160 within 4 years of propagation, without any decrease in vegetative propagation ability. A pilot production will now be carried out. (은 Inra/Elsevier, Paris.)
\end{abstract}

bulk vegetative propagation / cutting / stock-plant / Douglas-fir / Pseudostuga menziesii

Résumé - Le programme français d'amélioration génétique du Douglas génère des sources de graines très performantes (familles de demi ou de plein-frères) mais en quantité insuffisante pour être une source importante de matériel forestier de reboisement. La multiplication végétative en vrac (MVV) permet de prendre le relais en amplifiant ces lots de graines. Deux démarches complémentaires sont actuellement évaluées : sélection de familles pour leur aptitude à la MVV [31] et définition d'un parcours technique performant et économique pour l'élevage des pieds-mères de Douglas. À cette dernière fin, trois essais ont été menés entre 1992 et 1997 à l'Inra d'Orléans ct au Cemagref de Nogent/Vernisson comparant différentes ambiances d'élevage des pieds-mères, l'intérêt d'un

* Correspondance et tirés à part

E-mail : michel.verger@orleans.inra.fr 
complément d'éclairage artificiel, l'effet de la taille et le comportement des boutures terminales et intermédiaires. Le meilleur itinéraire consiste en une première récolte de boutures sur des pieds-mères âgés de 2 ans, élevés sur paillage plastique en sol de pépinière avec un ombrage blanc à $50 \%$. Dans ce cas, on a pu obtenir un coefficient de multiplication de 160 sur 4 années de récolte. Suite à ces essais, une production pilote de boutures de Douglas va être expérimentée à la pépinière administrative de Peyrat le Château afin de déterminer la faisabilité de la technique et de constituer un réseau de démonstration en forêt. (@) Inra/Elsevier, Paris.)

multiplication végétative en vrac / bouture / pied-mère / sapin de Douglas / Pseudostuga menziesii

\section{INTRODUCTION}

Le but d'un programme d'amélioration génétique est de sélectionner des génotypes apportant un gain sur des caractères d'intérêt sylvicole et de les diffuser à grande échelle sous forme de sorties variétales génératives (plants issus de graines) ou clonales (plants issus de boutures). Les avantages de ces dernières sont multiples : capture de la composante non additive de la variance génétique et donc gain génétique maximum, homogénéité de la production et raccourcissement des rotations, affranchissement des contraintes liées à la reproduction sexuée [20]. On considère deux types de sorties variétales clonales [33] :

- clonales au sens strict, dont le principe est de diffuser un ou quelques clones en mélange ;

- bulk qui correspondent à l'amplification par multiplication végétative en vrac (MVV) de matériel jeune (de quelques mois jusqu'à 4 ans selon les espèces), issu de lots de graines performants mais disponibles en faible quantité [34].

Le programme français d'amélioration du Douglas génère, sous la forme de familles de plein-frères, ce dernier type de matériel que seule la MVV pourrait actuellement valoriser [1]. Cependant les techniques de MVV actuellement mises en œuvre font que le prix des plants issus de boutures est supérieur à celui des plants issus de semis dans un rapport de 1 à $(1,5-3,5)[14,23,24]$ en fonction des espèces et des techniques. Il est donc important de définir un itinéraire de production, du pied-mère au plant en forêt, performant et économique. Le propos des essais décrits dans cet article est de définir un parcours optimal pour la première étape du processus, l'élevage des piedsmères, qui consiste à produire un nombre maximum de boutures aptes physiologiquement à s'enraciner et donnant des plants conformes aux normes de reboisement.

En fonction du degré de contrôle des paramètres environnementaux, on distingue différents modes de conduite des piedsmères, allant d'un élevage intensif à un élevage rustique.

L'élevage intensif des pieds-mères est réalisé sous serre et la plupart des paramètres environnementaux et agronomiques sont contrôlés. Les pieds-mères sont généralement renouvelés tous les ans (exemple : le Douglas aux États-Unis à la Weyerhauser Company [29]). L'élevage semi-intensif des pieds-mères se fait en serre non chauffée et/ou non éclairée. Les boutures sont le plus souvent récoltés sur des pieds-mères de deux ans. Afin d'augmenter le coefficient de multiplication (nombre de boutures enracinées par pied-mère), un bouturage en cascade (boutures récoltées sur des boutures en élevage) ou un bouturage réitéré (récolte de boutures répétée plusieurs années sur les mêmes pieds-mères) sont parfois réalisés (exemple : l'épicéa de Sitka en Grande-Bretagne $[23,25])$. Dans un élevage rustique, les pieds-mères sont élevés en bâche remplie de substrat ou directement en pleine terre. 
Pratiquement aucun paramètre climatique n'est contrôlé (exemple : le pin radiata en Nouvelle-Zélande $[11,26]$ ).

En France, dans le cadre du Groupement d'Intérêt Scientifique « variétés forestières améliorées », l'élevage intensif des piedsmères de Douglas a été étudié conjointement par l'Afocel, le Cemagref et l'Inra. Les résultats obtenus sont similaires à ceux de la Weyerhauser Company [3, 13]. Cependant, une première approche économique a montré que le coût de ce type d'élevage n'était pas compatible avec les modes de production de matériel forestier de reboisement en France : coût de la main-d'œuvre et du fonctionnement pour l'élevage d'un pied-mère de 40 francs pour 50 boutures prélevables (Généré, communication personnelle). Seul un élevage semi-intensif ou rustique pourrait donc être transférable chez les pépiniéristes forestiers. C'est là l'objet de notre étude.

\section{MATÉRIEL ET MÉTHODES}

Les principales caractéristiques des essais monés à l'Inra d'Orléans (essais 1 et 2) et au Cemagref de Nogent/Vernisson (essai 3) sont résumées dans le tableau I. La figure 1 indique le déroulement chronologique de ces différents essais.

\subsection{Origine génétique et élevage des pieds-mères}

Les graines utilisées proviennent d'un lot moyen du verger à graines de Bout (Allier) constitué d'arbres «plus », sélectionnés sur la vigueur et la forme dans des peuplements du Beaujolais. Elles ont été stratifiées pendant 4 à 5 semaines dans de la tourbe humide à $2{ }^{\circ} \mathrm{C}$. Le semis a été réalisé en mars 1992 (essai 1), mars 1993 (essai 2) et février 1993 (essai 3) en palet de tourbe Jiffy-7®, sous serre en verre chauffée (température minimum : $10^{\circ} \mathrm{C}$, température maximum : $25^{\circ} \mathrm{C}$ ) et sous lumière naturelle.

\subsubsection{Essais 1 et 2}

Les semis ont été repiqués en pépinière (sol de type sableux : >70\% de sable) en avril 1992 (essai 1) et avril 1993 (essai 2) sur un paillage de plastique noir (2 rangées par paillage) de $80 \mu \mathrm{m}$ d'épaisseur et de $1,25 \mathrm{~m}$ de large (distance entre pieds-mères : $0,35 \mathrm{~m}$ entre ligne et $0,25 \mathbf{m}$ sur la ligne). Les pieds-mères de l'essai 1 ont été élevés sous un tunnel de $13 \mathrm{~m}$ de long, $4,5 \mathrm{~m}$ de large et $2 \mathrm{~m}$ de haut au centre, recouvert d'une toile climatique blanche assurant $50 \%$ d'ombrage. Elle a été retirée durant l'hiver 1994-95. Les pieds-mères de cet essai ont été récoltés en 1994, 1995, 1996 et 1997. Les picdsmères de l'essai 2 ont été élevés dans différentes ambiances décrites au paragraphe modalités expérimentales. Ils ont été récoltés en 1995, 1996 et 1997. Pour les pieds-mères des deux essais, une irrigation fertilisante (équilibre 15.11.15) a été apportée quotidiennement par un système de goutte à goutte d'avril à mi-septembre. Après la première récolte de boutures puis tous les ans, les pieds-mères ont tous subi une taille uniforme consistant à étêter l'axe principal à $45 \mathrm{~cm}$ de haut et à ramener les latéraux à $1,5 \mathrm{~cm}$ du tronc.

\subsubsection{Essai 3}

À partir d'avril 1993, les pieds-mères ont été élevés en conteneur de $3 \mathrm{~L}$ dans un mélange tourbe/sable/écorce (4- 3-3 v/v) comportant une combinaison d'engrais retard 3-4 mois 18-6-12 $\left(0,5 \mathrm{~kg} \mathrm{~m}^{-3}\right)$ et $12-14$ mois $15-8-11+2 \mathrm{MgO}$ $\left(3,5 \mathrm{~kg} \mathrm{~m}^{-3}\right)$. L'essai a été installé à la densité de 20 plants $\mathrm{m}^{-2}$ sous tunnel de $18 \mathrm{~m}$ de long, $4,5 \mathrm{~m}$ de large et $2 \mathrm{~m}$ de haut au centre, recouvert d'une toile climatique blanche (ombrage à $50 \%$ ). L'irrigation a été effectuéc par un système de goutte à goutte. Chaque année, une fertilisation de fond a été apportée à raison de $150 \mathrm{~g} \mathrm{~m}^{-2}$ d'engrais retard $12 / 14$ mois $15-8-11+2 \mathrm{MgO}$ mis en surfaçage. Les pieds-mères ont été récoltés en 1994, 1995 et 1996.

\subsection{Bouturage}

Ont été considérées comme des boutures prélevables toutes les pousses parfaitement droites de $8 \mathrm{~cm}$ de long, de diamètre compris entre 1,5 et $3 \mathrm{~mm}$, avec un bourgeon terminal (boutures terminales) ou sans bourgeon terminal (boutures intermédiaires). La récolte au sécateur des boutures a été réalisée à la mi-janvier (essai 3) ou à la mi-février (essais 1 et 2). Elles ont été conser- 


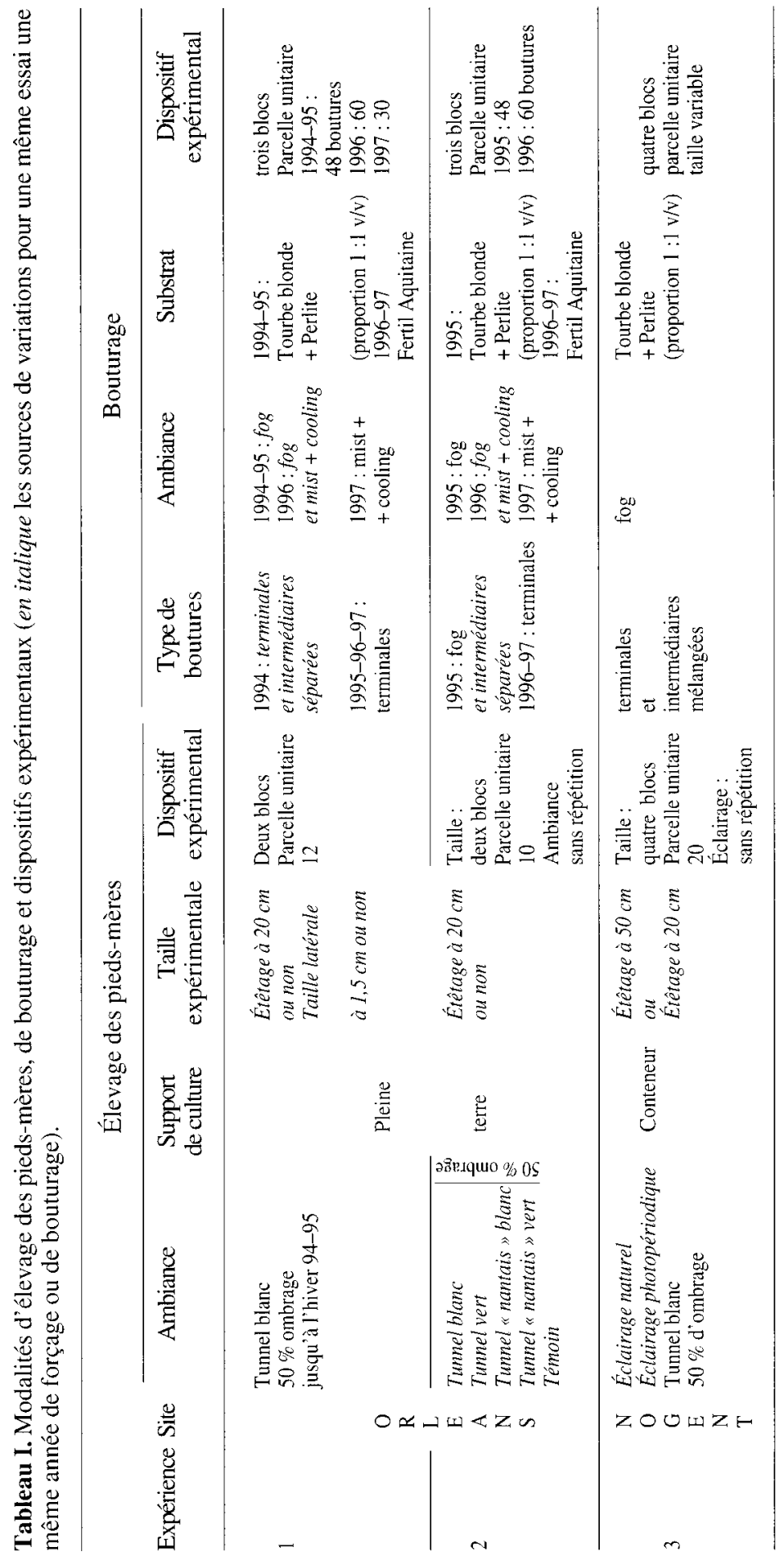




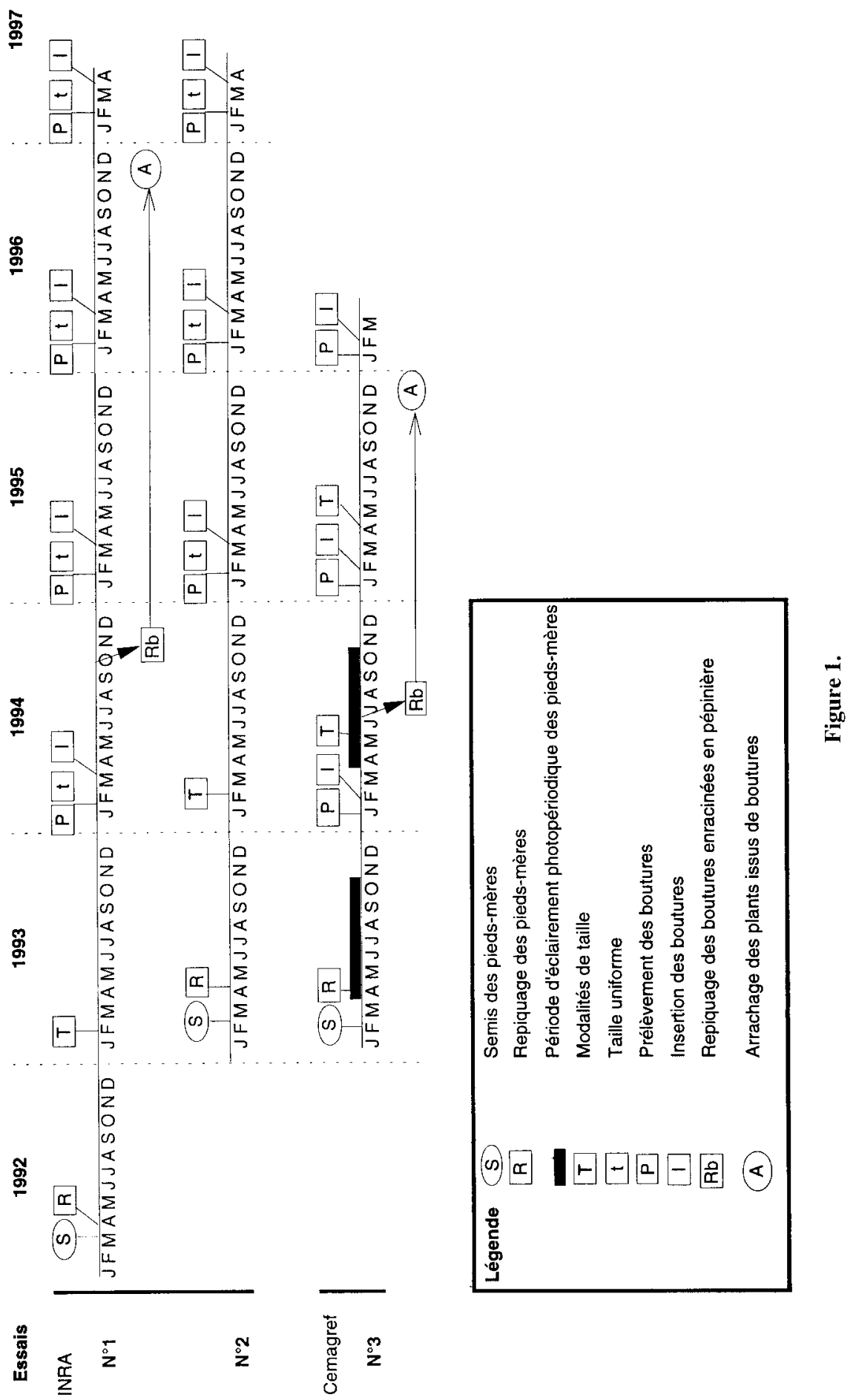


vées systématiquement un mois en chambre froide entre $0^{\circ} \mathrm{C}$ et $2{ }^{\circ} \mathrm{C}$ en sacs plastiques hermétiques et à l'obscurité. Juste avant l'insertion, la base des boutures, débarrassée des aiguilles sur $2 \mathrm{~cm}$ environ, a été trempée dans une solution aqueuse à $1000 \mathrm{ppm}$ d'acide indole butyrique (AIB) pendant $10 \mathrm{~s}$. Les boutures ont ensuite été insérées dans des plaques alvéolées en polystyrène thermoformé $(53 \mathrm{~cm} \times 30 \mathrm{~cm}$ et $7,5 \mathrm{~cm}$ de profondeur, 112 alvéoles tronconiques de $48 \mathrm{~cm}^{3}$, 700 boutures $\mathrm{m}^{-2}$ ) ou dans des caisses plastiques ajourées $\left(60 \times 40 \times 13 \mathrm{~cm}, 250\right.$ boutures $\left.\mathrm{m}^{-2}\right)$ pour le bouturage des essais 1 et 2 en 1996 et 1997. Au cours des différentes campagnes de bouturage, deux substrats ont été alternativement utilisés : mélange de tourbe blonde irlandaise et de perlite ( $\mathrm{G} 40)$ en proportion $1-1(\mathrm{v} / \mathrm{v})$ ou substrat commercial Fertil Aquitaine spécial multiplication ${ }^{\circledR}$ pour le bouturage des essais 1 et 2 en 1996 et 1997. Les températures de fond et d'ambiance ont été supérieures respectivement à $18^{\circ} \mathrm{C}$ et $10^{\circ} \mathrm{C}$. En début de phase de bouturage, l'hygrométrie a été maintenue aux environs de 85-90\% grâce à l'utilisation de brouillard artificiel (fog) ou d'un cooling system plus un arrosage intermittent (mist) pour les essais 1 et 2 en 1996 et 1997. Elle a ensuite été réduite progressivement dès l'apparition des premières racines. Des traitements hebdomadaires contre le Botrytis ont été réalisés durant toute la phase de bouturage en alternant les matières actives.

Pour les essais 1 et 2 , seul un échantillon aléatoire des boutures récoltées par parcelle unitaire d'élevage des pieds-mères a été inséré. Pour l'essai 3 , l'ensemble des boutures récoltées a été inséré.

\section{3. Élevage des boutures en pépinière}

En octobre 1994, les boutures enracinées de l'essai 1 ont été repiquées mécaniquement (ćcartements : $20 \mathrm{~cm}$ sur la ligne et $60 \mathrm{~cm}$ entre les lignes) à la pépinière de l'Inra d'Orléans après désinfection du sol au bromure de méthyle. Un cernage horizontal a été effectué en juillet 1996. Les boutures ont été arrachées durant l'hiver 1996-97.

En juin 1994, les boutures enracinées de l'essai 3 ont été repiquées manuellement (écartements : $10 \mathrm{~cm}$ sur la ligne et $25 \mathrm{~cm}$ entre les lignes) à la pépinière de Peyrat-le-Château dans la Haute-Vienne. Un cernage vertical a été réalisé fin août 1995 et l'arrachage des plants a été effectué durant l'hiver 1995-96.

\subsection{Modalités expérimentales}

Un certain nombre de facteurs, tous connus pour améliorer la production de boutures et/ou l'aptitude à l'enracinement de ces boutures, ont été expérimentés sur les pieds-mères :

- la taille (essais 1, 2 et 3) qui peut arrêter ou retarder les effets du vieillissement $[15,16$, $21]$ voire permettre le rajeunissement des pieds-mères $[6,12]$;

- l'ambiance d'élevage (essai 2);

- l'apport d'éclairage additionnel (essai 3).

D'autres paramètres ont été secondairement testés : effet du type de boutures récoltées et de l'ambiance de bouturage.

\subsubsection{Essai 1}

Le but de cet essai est de comparer quatre modalités de taille des pieds-mères, effectuées en février 1993 et résultant de la combinaison d'un étêtage (pas d'étêtage ou étêtage à $20 \mathrm{~cm}$ de haut) et d'une taille des rameaux latéraux (pas de taille ou taille à $1,5 \mathrm{~cm}$ du tronc).

\subsubsection{Essai 2}

Dans cet cssai, est évalué le comportement de pieds-mères élevés dans cinq ambiances différentes (uniquement trois pour la campagne de bouturage de 1997) et ayant subi deux types de taille : étêtage à $20 \mathrm{~cm}$ de haut réalisé en février 1994 ou aucune taille. Ils ont été élevés :

- sous tunnel (mêmes dimensions que pour l'essai 1), recouvert de toile climatique soit blanche soit verte assurant $50 \%$ d'ombrage ;

- sous tunncl de type « nantais » $(0,5 \mathrm{~m}$ de haut au centre, $1 \mathrm{~m}$ de large), recouvert également de toile climatique soit blanche soit verte assurant $50 \%$ d'ombrage ; pour ce type de tunnel, la toile climatique a été retirée en début de seconde année de végétation, les pieds-mères l'atteignant. ;

- sur paillage plastique sans toile climatique (= témoin).

En 1997, compte tenu des résultats antérieurs, les pieds-mères des deux modalités « toile climatique verte » n'ont plus été bouturés.

\subsubsection{Essai 3}

Le but de cet essai est de comparer, pour des pieds-mères élevés en conteneur, l'effet de deux types d'éclairage croisés avec deux types d'étê- 
tage : étêtage à 50 ou $20 \mathrm{~cm}$ de haut réalisé après le prélèvement des premières boutures (mai 1994). Ces tailles ont été appliquées de nouveau en avril 95.

La moitié des pieds-mères a été élevée sous un éclairage additionnel photopériodique (lampes Philips SL 18 R 18 W) d'avril à octobre (en 1993 et en 1994), de $5 \mathrm{~h}$ à $23 \mathrm{~h}$, lorsque la lumière naturelle descendait en dessous de $10 \mathrm{~W} \mathrm{~m}^{-2}$. L'autre moitié a été élevée sous lumière naturelle.

\subsection{Mesures}

Les mesures portent sur le nombre de boutures terminales et/ou intermédiaires récoltées par pied-mère (essais 2 et 3 ) ou par parcelle unitaire d'élevage des pieds-mères (essai 1) et sur le pourcentage d'enracinement. Une bouture est considérée comme enracinée si elle présente au moins une racine de $2 \mathrm{~mm}$ de longueur. Les relevés d'enracinement sont effectués au moins 5 mois après l'insertion des boutures. Le coefficient de multiplication est calculé, produit du nombre de boutures récoltées par pied-mère et du pourcentage d'enracinement. Une saison (essai 3, bouturage 1993) ou deux saisons (essai 1, bouturage 1993) après repiquage, le pourcentage de reprise des plants repiqués a été calculé de même que le taux de plants commercialisables selon les normes de qualité FFN [10].

\subsection{Analyses statistiques}

Les analyses statistiques sont réalisées avec le logiciel Statgraphics ${ }^{\mathrm{TM}}$. Elles consistent en analyses de la variance multifacteurs en modèle fixe avec contrôle de l'hétérogénéité du milieu par des blocs. Les modèles d'analyse de variance sont donnés dans les tableaux $I I, I I I$ et $I V$. Lorsqu'il y a des différences significatives entre modalités et en l'absence d'effet d'interaction significatif, le test de comparaison de moyennes de Tukey au seuil d'erreur de $5 \%$ est utilisé.

\section{RÉSULTATS}

\subsection{Essai 1 (tableau II)}

En moyenne, 192 boutures, terminales et intermédiaires confondues, ont été récol- tées par pied-mère sur quatre campagnes. Les boutures intermédiaires n'ont pu être récoltées qu'en 1994, les rameaux latéraux n'étant pas les années suivantes suffisamment allongés. Il n'apparaît d'effet significatif des modalités de taille que sur le nombre de boutures intermédiaires récoltées par pied-mère en 1994 et sur le nombre de boutures terminales récoltées par piedmère en 1996.

Il n'y a pas d'effet de la taille sur le pourcentage d'enracinement. Les pourcentages d'enracinement des boutures terminales et intermédiaires en 1994 sont tout à fait similaires. Notons que le pourcentage d'enracinement moyen est plus élevé en 1995 et en 1997 qu'en 1994 alors que les pieds-mères sont plus âgés. Le pourcentage d'enracinement est très mauvais en 1996 pour l'ambiance fog alors qu'il est excellent ( $76 \%$ ) pour la seule modalité (témoin) bouturée en ambiance mist.

Il existe un effet significatif de la taille sur le coefficient de multiplication sauf en 1996.

Tous traitements confondus, le taux de survie après deux ans d'élevage en pépinière des plants repiqués en 1994 est de $84 \%$ et le taux de plants conformes aux normes de reboisement FFN par rapport aux boutures vivantes est de $72 \%$.

\subsection{Essai 2 (tableau III)}

En moyenne, 169 boutures, terminales et intermédiaires confondues, ont été récoltées par pied-mère sur trois campagnes. Les boutures intermédiaires n'ont pu être récoltées que dans deux ambiances (les grands tunnels), les rameaux latéraux n'étant pas suffisamment allongés dans les autres ambiances. On note un effet hautement significatif de l'ambiance d'élevage des pieds-mères sur la production de boutures. Il n'existe d'effet de la taille des pieds-mères que sur le nombre de boutures intermédiaires récoltées en 1995 et par contre coup sur la 


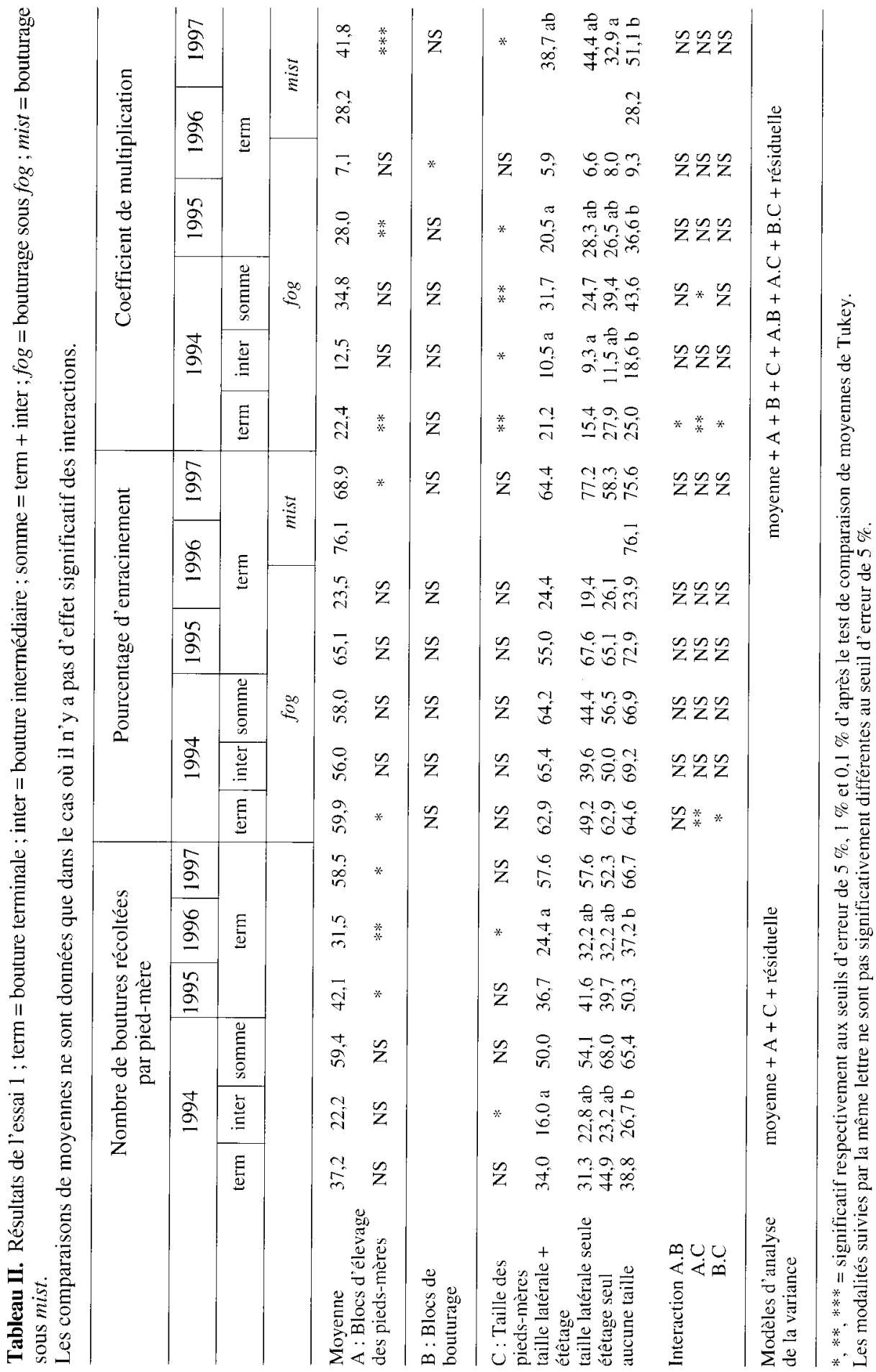




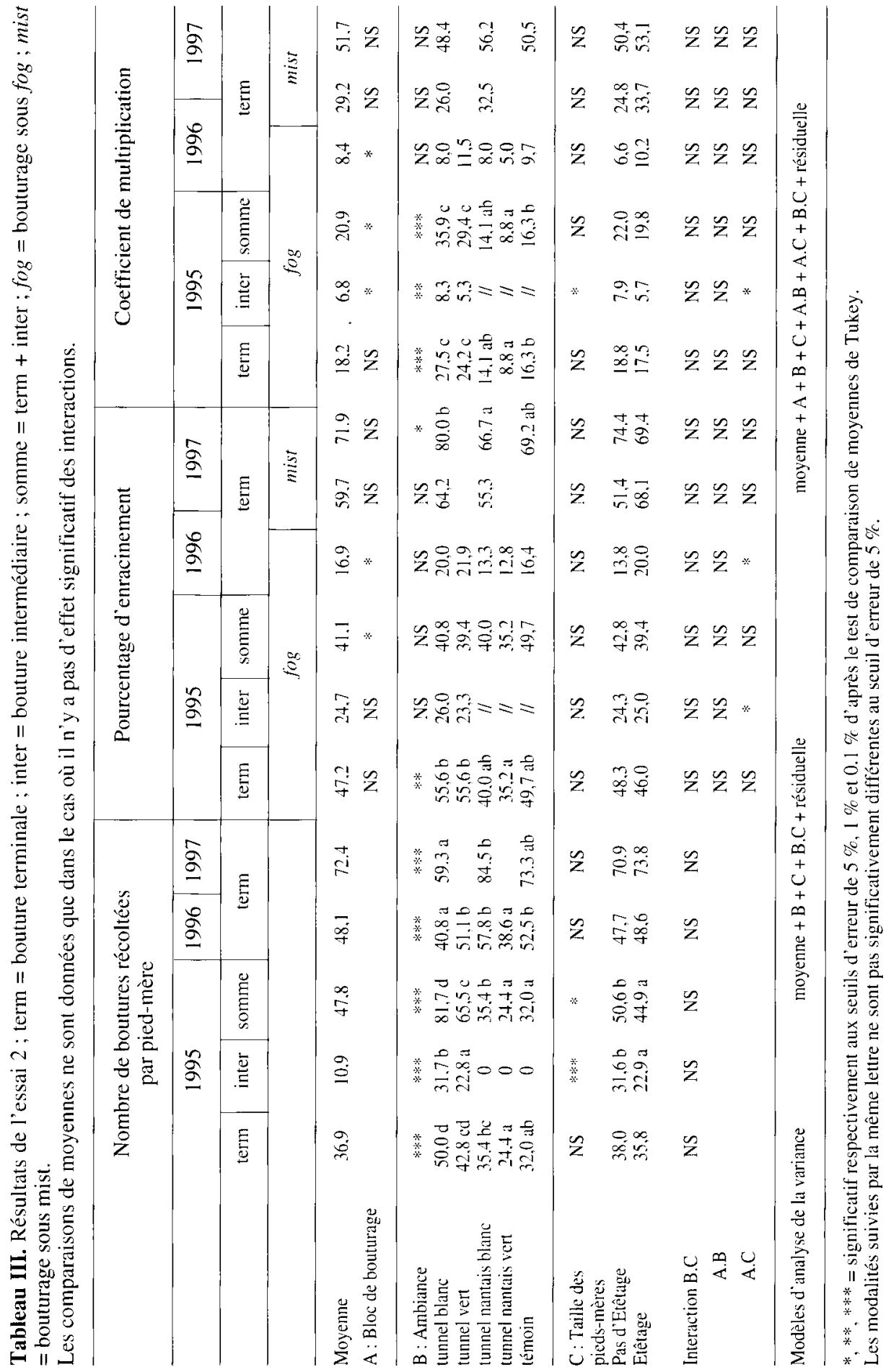


somme "terminales plus intermédiaires » pour cette même année.

Il n'existe d'effet significatif de l'ambiance d'élevage des pieds-mères sur le pourcentage d'enracinement qu'en 1995 et 1997 pour les boutures terminales. Il n'y a pas d'effet de la taille sur l'enracinement des boutures. Le pourcentage d'enracinement semble moins bon pour les boutures intermédiaires que pour les boutures terminales. Comme dans l'essai 1 , les résultats sous fog en 1996 sont très mauvais alors qu'ils peuvent dépasser $60 \%$ sous mist.

L'ambiance agit significativement sur le coefficient de multiplication uniquement en 1995. La taille n'agit que sur la composante bouture intermédiaire du coefficient de multiplication en 1995.

\subsection{Essai 3 (tableau IV)}

En moyenne, on a pu récolter 58 boutures, terminales et intermédiaires confondues, par pied-mère sur trois campagnes soit 51 avec un éclairage naturel et 65 avec le complément photopériodique. La taille n'agit pas sur le nombre de boutures prélevées. L'éclairage photopériodique améliore significativement le nombre de boutures terminales récoltées les deux premières années. Il n'augmente le nombre de boutures intermédiaires qu'en première année. En 1996 , l'éclairage n'agit plus sur le nombre de boutures prélevées.

Le pourcentage d'enracinement n'est pas influencé par le type d'étêtage. En revanche l'éclairage photopériodique diminue significativement le pourcentage d'enracinement en 1994 et 1995. En 1996 son effet s'annule.

Le coefficient de multiplication n'est pas influencé par le type d'étêtage. Le type d'éclairage agit significativement sur le coefficient de multiplication uniquement la première année de bouturage.

Tous traitements confondus, le taux de survie après un an d'élevage en pépinière des plants repiqués en 1994 est de $83 \%$ et le taux de plants conformes aux normes de reboisement FFN par rapport aux boutures vivantes est de $68 \%$.

\section{DISCUSSION}

\subsection{Pourcentage d'enracinement}

Les pourcentages d'enracinement obtenus lors des différentes expérimentations sont en concordance avec les résultats trouvés dans la bibliographie sur le bouturage du Douglas avec des techniques et des piedsmères d'âges similaires : entre 68 et $78 \%$ pour des pieds-mères de 2 à 4 ans [7], entre 56 et $68 \%$ pour des pieds-mères de 4 ans [4], $40 \%$ pour des pieds-mères de 5 ans [27]. Des pourcentages d'enracinement de $100 \%$ peuvent cependant être observés avec des pieds-mères plus âgés (jusqu'à 9 ans) mais au niveau individu (clone) et non plus population [4]. Les résultats obtenus avec des pieds-mères plus jeunes ( 1 an) sont également meilleurs : jusqu'à $90 \%$ [29].

Le vieillissement des pieds-mères peut (essai 3) ou non (essais 1 et 2) s'accompagner d'une diminution de l'aptitude à l'enracinement. Il a déjà été observé sur Douglas [9] que le vieillissement des pieds-mères pouvait se traduire par une augmentation de l'aptitude à l'enracinement, attribuable à l'effet bénéfique de la taille des pieds-mères, à l'amélioration des techniques de bouturage... et du savoir-faire [8].

Les bons résultats obtenus sous mist en 1996 et 1997 (essais I et 2) montrent également que le choix de l'ambiance de bouturage peut être crucial pour la réussite du bouturage du Douglas. On peut penser que l'ambiance initialement choisie (fog) n'était pas la meilleure, notamment du fait de la difficulté à maîtriser les excès de température (des températures proches de $40^{\circ} \mathrm{C}$ ont été enregistrées) ou que les conditions optimales d'enracinement changent avec le vieillissement des pieds-mères. Une interaction entre l'ambiance de bouturage et 


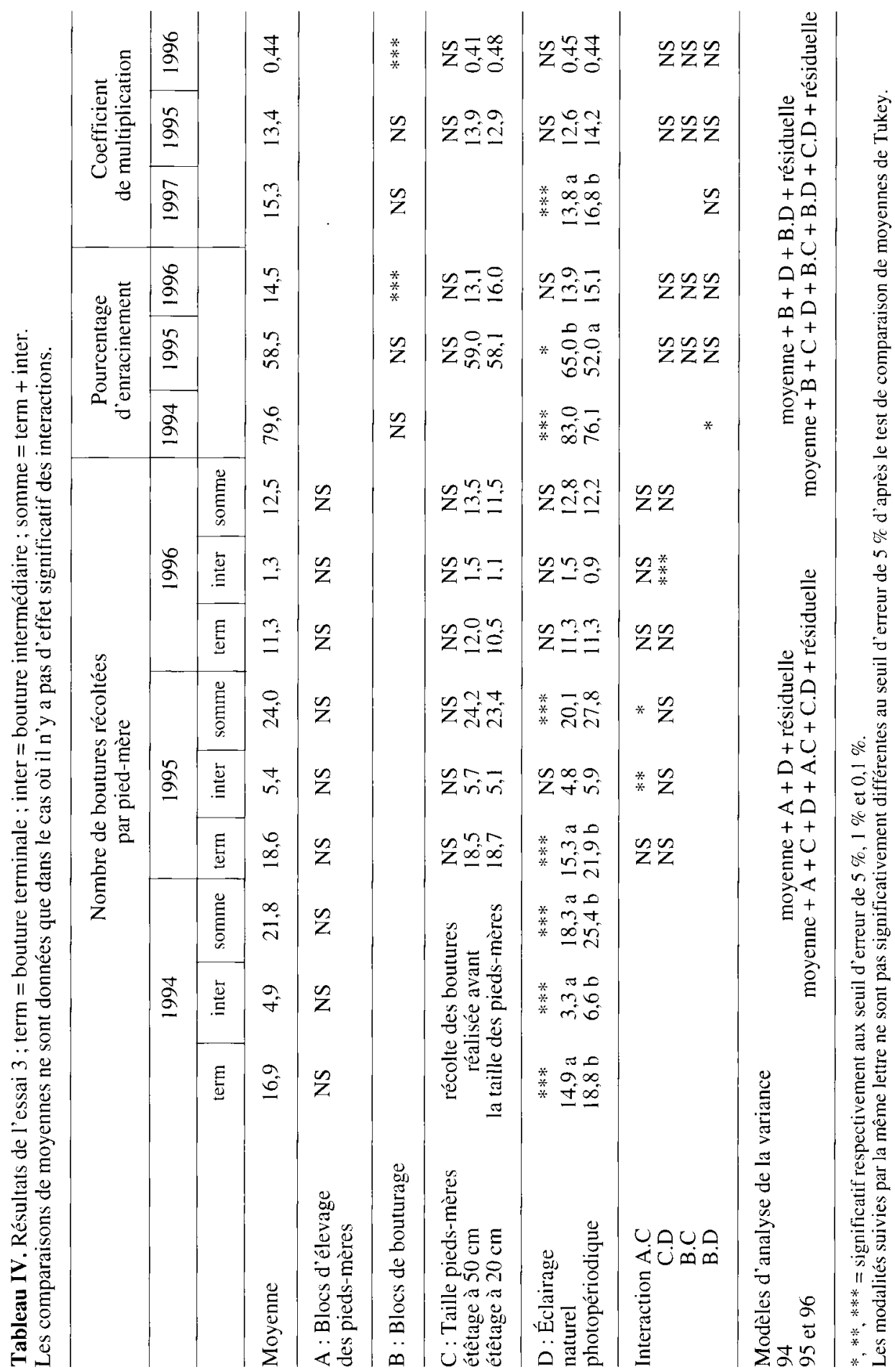


l'année climatique, défavorable au bouturage sous fog pourrait également expliquer ces résultats.

\subsection{Effet du type de boutures}

Dans les essais décrits, les bouiures intermédiaires ont été récoltées systématiquement (essai 3) ou dans quelques modalités (essais 1 et 2) lorsque les latéraux se sont suffisamment allongés. On voit dans le dernier cas qu'elles ne sont présentes que dans les ambiances sous grand tunnel et uniquement l'année après la réalisation des modalités de taille. Les années suivantes, du fait de la taille uniforme, elles n'ont pas pu se développer.

Pour des pieds-mères conduits dans les mêmes conditions, le pourcentage d'enracinement des boutures terminales et intermédiaires peut être similaire (essai 1) ou très différent (du simple au double dans l'essai 2). Des résultats semblables avaient déjà été trouvés avec des pieds-mères de différentes origines génétiques [3]. De même, la bibliographie sur le bouturage des ligneux est riche en semblables contradictions entre des auteurs qui estiment que l'on peut, pour les conifères comme pour les feuillus, utiliser indifféremment les deux types de boutures [32] et ceux qui estiment que les boutures intermédiaires donnent systématiquement de mauvais ou de moins bons résultats ([5] sur Douglas, [22] sur mélèze hybride). On peut donc plutôt parler d'instabilité de la réponse des boutures intermédiaires que d'une inaptitude ou d'une mauvaise aptitude chronique à l'enracinement. L'absence du bourgeon terminal dont on reconnaît le rôle important dans l'enracinement adventif [19] et particulièrement pour le Douglas $[2,30]$ explique certainement cette instabilité.

De façon pratique et du fait de cette instabilité, il peut être conseillé de n'enraciner les boutures intermédiaires que lorsque leur nombre le justifie et ce afin d'augmenter le coefficient de multiplication. Dans l'essai
1, les boutures intermédiaires représentent en moyenne $35 \%$ du coefficient de multiplication 1994 et $20 \%$ dans l'essai 2 . On pourrait également favoriser sur le pied-mère la production de boutures terminales grâce à des tailles plus hautes qui permettrait le démarrage d'un plus grand nombre de ramifications.

\subsection{Effet de l'apport de lumière sur les pieds-mères}

Les résultats obtenus avec l'apport de lumière sur les pieds-mères montrent un effet positif de l'éclairage photopériodique sur le nombre de boutures terminales et la somme «terminales plus intermédiaires » prélevées. Ce résultat se retrouve chez d'autres espèces comme le mélèze hybride pour lequel la lumière artificielle stimule la branchaison et le rendement en boutures [28]. Cependant cet effet positif ne perdure pas au-delà de la campagne de bouturage qui suit l'arrêt de l'éclairage. L'éclairage complémentaire devrait sans doute fonctionner tous les ans afin d'apporter un gain soutenu de boutures prélevables.

De plus l'enracinement des boutures récoltées sur des pieds-mères ayant subi un éclairage naturel est meilleur ou identique à celui des boutures récoltées sur des piedsmères ayant reçu un complément photopériodique. La diminution de l'aptitude à l'enracinement observée avec l'augmentation de l'intensité d'éclairage des piedsmères ou avec l'allongement de la photopériode a déjà été mentionnée sur le pin sylvestre $[17,18]$ et le mélèze hybride [23]. Il en ressort que le gain sur le coefficient de multiplication est très faible $(4,6)$ et que l'on peut s'interroger sur l'intérêt économique de cette technique dans le cadre de la MVV du Douglas. 


\subsection{Effet de la taille des pieds-mères}

Dans les essais décrits, on constate que les tailles expérimentées n'agissent jamais sur le pourcentage d'enracinement. En revanche, elles peuvent agir sur le nombre de boutures prélevables et sur le coefficient de multiplication (essais 1 et 2), même plusieurs années après une taille sévère, réitérée plusieurs années de suite et donc susceptible de réuniformiser les pieds-mères (effet significatif de la taille sur le coefficient de multiplication en 1997 soit 4 ans après son application pour l'essai 1).

\subsection{Effet de l'ambiance d'élevage et du support de culture des pieds-mères}

Différentes ambiances ont été testées, allant de l'élevage sur paillage plastique en sol de pépinière jusqu'à l'élevage en conteneur sous ombrière avec apport d'éclairage photopériodique. Il apparaît que le coefficient de multiplication n'est pas meilleur avec les ambiances les plus sophistiquées. C'est la modalité « témoin » de l'essai 1 qui a donné le meilleur coefficient de multiplication : 160 (sur 4 années de récolte), en considérant le résultat sous mist pour le bouturage de 1996. L'ambiance « tunnel nantais blanc $\gg$ de l'expérimentation 2 (coefficient de multiplication de 103, en considérant les résultats sous mist en 1996, sur 3 années de récolte) offre également un très bon compromis entre rendement en boutures enracinées et coûts d'installation.

L'élevage en conteneur donne des résultats inférieurs. Il permet en revanche une première récolte de boutures après une seule année d'élevage. Il pourrait donc être réservé à une amplification rapide de sources de graines, plus dans le cadre des programmes d'amélioration génétique que dans celui de la production de matériel forestier de reboisement.

\section{CONCLUSION ET PERSPECTIVES}

Pour le Douglas, la fourniture en masse de matériel forestier de reboisement génétiquement performant passera soit par des techniques de vergers à graines gérés intensivement, soit par des techniques de multiplication végétative : bouturage horticole ou embryogenèse somatique. Pour être compétitive par rapport aux vitro-méthodes, susceptibles d'avoir un pouvoir d'amplification plus performant, la multiplication végétative en vrac doit être compatible avec des coûts de production très faibles et une technicité réduite. Actuellement, on peut voir converger deux démarches allant dans ce sens : la sélection génétique pour l'aptitude à la multiplication végétative en vrac de familles performantes d'un point de vue sylvicole [31] et la simplification des méthodes d'élevage des pieds-mères.

Les essais décrits dans cet article montrent que des techniques d'élevage rustique peuvent être des alternatives intéressantes à l'élevage intensif des pieds-mères. Des gains importants peuvent être certainement encore obtenus, notamment en recherchant de meilleures ambiances de bouturage. De même des techniques de pépinière plus appropriées aux plants issus de boutures (dates de repiquage, densité, fertilisation...) pourraient certainement encore améliorer le rendement en plants conformes aux normes FFN. Certains points restent encore à expérimenter, le plus important étant le comportement en pépinière puis en forêt de plants issus de pieds-mères âgés de plus de 2 ans.

Afin d'étudier à un niveau plus opérationnel une filière de production de plants de Douglas issus de multiplication végétative en vrac, une production pilote regroupant I'Inra, le Cemagref et la pépinière administrative de Peyrat le Château est expérimentée. Le but de cette production est notamment d'associer les deux démarches: sélection génétique de matériel performant pour la multiplication végétative en vrac et 
simplification des méthodes d'élevage des pieds-mères. Par ailleurs le matériel végétal produit permettra de constituer un réseau de démonstration en forêt qui validera ou non l'intérêt d'une filière de production de plants de Douglas issus de multiplication végétative en vrac.

\section{REMERCIEMENTS}

Les auteurs remercient tout le personnel technique associé au projet : les équipes « Multiplication végétative » et « Expérimentations en pépinière » de l'Inra d'Orléans, « Pépinière » de la division RGPF du Cemagref de Nogent/Vernisson et le personnel de la pépinière administrative de Peyrat-le-Château.

L'étude a été réalisée grâce à un financement du Groupement d'Intérêt Scientifique (GIS) «Variétés forestières améliorées » et du contrat CEE (DG XII) « Conifères à croissance rapide » (AIR $n{ }^{\circ} \mathrm{CT} 920143$ ).

Tous nos remerciements également à Virginic Pellicer, Jean-Charles Bastien, Marc BonnetMasimbert, Daniel Cornu, Jcan-Yves Gautry, Hervé Le Bouler et Nicolas Schermann pour les améliorations qu'ils ont apportées au manuscrit ainsi qu'aux correcteurs des Annales des Sciences Forestières.

\section{RÉFÉRENCES}

[1] Bastien J.C., Michaud D., Héoïs B., Douglas, Forêt Entreprise 96 (1994) 66-69.

(2) Bhella H.S., Roberts A.N., Bud and cambial activity in Douglas-fir as related to stem cutting rootability, For. Sci. 21 (1975) 269-275.

[3] Bidaud N., Cazet M., Gautry J.Y., Verger M., Résultats préliminaires sur la multiplication végétative « Bulk » du Douglas, in : Afocel (éd.) Production de variétés génétiquement améliorées d'espèces forestières à croissance rapide, Symposium Bordeaux France, 1992, t. II, 321-330.

[4] Black D.K., The influence of shoot origin on the rooting of Douglas-fir stem cuttings, Comb. Proc. Int. Plant Propag. Soc. 22 (1972) 142-147.

[5] Brix H., Barker H., Rooting studies of Douglas-fir cuttings, Can. For. Ser. Pac. For. Res. Cent. Rep., Victoria, Br. Col., BC-X-87, 1973, 45 p.

[6] Chaperon H., Maturation et bouturage des arbres forestiers. Afocel, Études et Recherches 12 (1979) 19-31.
[7] Copes D.L., Influence of rooting media on root structure and rooting percentage of Douglas-fir cuttings, Silvae Genet. 26 (1977) 102-106.

[8] Copes D.L., Effects of annual crown pruning and serial propagation on rooting of stem cuttings from Douglas-fir, Can. J. For. Res. 13 (1983) 419-424.

[9] Copes D.L., Effects of long-term pruning, meris tem origin and branch order on the rooting of Douglas-fir stem cuttings, Can. J. For. Res. 22 (1992) $1888-1894$

[10] Derf (Paris), ONF (Paris), Cemagref (Nogent/Vernisson) et al., Réussir la forêt, contrôle et réception des travaux. Derf, Paris, 1990,61 p.

[11] Faulds T., Dibley M.J., Growing radiata pine from juvenile cuttings. What's New in Forest Research 176, 1989, $4 \mathrm{p}$.

[12] Franclet A., Manipulation des pieds-mères et amélioration de la qualité des boutures. Afocel, Études et Recherches 8, 1977, 20 p.

[13] Gautry J.Y., La multiplication par bouturage en vrac : une méthode pour accélérer la diffusion des variétés de Douglas, Information-Forêt 517 (1995) 271-286.

[14] Graham J., Gill S., Comparisons of production costs and genetic benefits of transplants and rooted cuttings of Picea sitchensis, Forestry (Oxf.) 56 (1983) 61-73.

[15] Hackett W.P., Donor Plant Maturation and Adventitious Root Formation. in : Davis T.D., Haissig B.E., Sankhla N. (Eds.), Adventitious Root Formation in Cuttings, Dioscorides press, Portland, Oregon, 1988, 11-28.

[16] Haines R.J, Walker S.M., Copley T.R., Morphology and rooting of shoots developing in response to decapitation and pruning of Caribbean pine, New For. 7 (1993) 133-141.

[17] Hansen J., Ernsten A., Seasonal changes in adventitious root formation in hypocotyl cuttings of Pinus sylvestris L. : Influence of photoperiod during stock plant growth and of indolbutyric acid treatment of cuttings, Physiol. Plant. 54 (1982) 99-106.

[18] Hansen J., Strömquist L.-H., Ericsson A., Influence of the irradiance on carbohydrate content and rooting of cuttings of pine seedlings (Pinus sylvestris L.). Plant Physiol. (Bethesda) 61 (1978) 975-979.

[19] Hartmann H.T., Kester D.E., Plant Propagation. Principles and Practices, Prentice Hall international, New Jersey, 1990, $727 p$

[20] Libby W.J., The clonal option, Norsk Institut for Skogforskning, Norw. For. Res. Inst., 1983, 32 p.

[21] Libby W.J., Brown A.G., Fielding J.M., Effects of hedging Radiata pine on production, rooting, and early growth of cuttings, N. Z. J. For. Sci. 2 (1972) 263-283.

[22] Mason W.L., Vegetative propagation of conifers using stem cuttings. II Hybrid Larch., For. Comm. Res. Inf., Note 91/84/Siln, 1984, 3 p. 
[23] Mason W.L., Reducing the cost of Sitka Spruce cuttings. in : Rook D.A. (Ed), Super Sitka for the 90s, For. Comm. Bull. 103 (1992) 25-41.

[24] Mason W.L., Harper W.C.G., Forest use of improved Sitka Spruce cuttings, For. Comm. Res. Inf., Note $119 / 87 /$ SILN, 1987,4 p.

[25] Mason W.L., Jinks R.L., Vegetative propagation. in : Aldhous J.R., Mason W.L. (Eds.), Forest Nursery Practice, For. Comm. Bull. 111 (1994) 135-147.

[26] Menzies M.I., Faulds T., Dibley M.J., Vegetative propagation of juvenile Radiata Pine. in : Workshop on growing radiata pine from cuttings, FRI bulletin 135, N. Z. For. Res. Inst., (1988) 109-129.

[27] Myers J.F., Howe G.E., Vegetative propagation of rocky mountain Douglas-fir by stem cuttings, Tree Planters' Notes 41 (1990) 3-6.

[28] Pellicer V., Cazet M., Verger M., Rivière L.M., Effect of stock plant lighting on bulk vegetative propagation of hybrid Larch (Larix $x$ eurolepis Henry), For. Ecol. Manag. (1998) 323-332.

[29] Ritchie G.A., Production of Douglas-fir, Pseudostuga menziesii (Mirb.) Franco, rooted cuttings for reforestation by Weyerhaeuser Company, Comb. Proc. Int. Plant Propag. Soc. 43 (1993) 284-288.

[30| Roberts A.N., Fuchigami L.H., Seasonal changes in auxin effect on rooting of Douglas-fir stem cuttings as related to bud activity, Physiol. Plant. 28 (1973) 215-221.

131] Schermann N., Verger M., Bastien J.C., Sélection de familles de Douglas (Pseudotsuga menziesii) pour leur aptitude au bouturage : conséquences pour la diffusion de variétés améliorées, Ann. Sci. For. 53 (1996) 1113-1126.

[32] Thompson D.G., Current state-of-the-art of rooting cuttings and a view to the future, in : Afocel, (éd.), Production de variétés génétiquement améliorées d'espèces forestières à croissance rapide, Symposium Bordeaux, France, t. II, 1992, 333-349.

[33] Verger M., Plants forestiers génétiquement améliorés : production par multiplication végétative, Forêt Entreprise 114 (1997) 55-60.

[34] Verger M., Pâques L.E., Multiplication végćtative du Mélèze hybride (Larix $x$ eurolepis Henry) par multiplication végétative en vrac, Ann. Sci. For. 50 (1993) 205-215. 